\title{
(- OPEN ACCESS \\ Impact of surgical timing of postoperative ocular motility in orbital blowout fractures
}

\author{
Yukito Yamanaka, ${ }^{1}$ Akihide Watanabe, ${ }_{1}^{1}$ Chie Sotozono, ${ }^{1}$ Shigeru Kinoshita ${ }^{2}$
}

'Department of Ophthalmology, Kyoto Prefectural University of Medicine, Graduate School of Medicine, Kyoto, Japan ${ }^{2}$ Department of Frontier Medical Science and Technology for Ophthalmology, Kyoto Prefectural University of Medicine, Kyoto, Japan

Correspondence to Dr Akihide Watanabe, Department of Ophthalmology, Kyoto Prefectural University of Medicine, Graduate School of Medicine, 465 Kajii cho, Hirokoji agaru, Kawaramachi dori, Kamigyo ku, Kyoto 602 0841, Japan; awatanab@koto. kpu-m.ac.jp

Received 15 February 2017 Revised 8 June 2017

Accepted 16 June 2017 Published Online First 25 July 2017
Check for updates

To cite: Yamanaka $Y$, Watanabe A, Sotozono C, et al. Br J Ophthalmol 2018:102:398-403.

\section{ABSTRACT}

Purpose To investigate the surgical timing postinjury in regard to ocular motility in patients with orbital-floor blowout fractures.

Methods This study involved 197 eyes (92 right eyes and 105 left eyes) of 197 patients (154 males and 43 females, mean age: 29.0 years, range: $7-85$ years) with pure orbital blowout fractures. All patients underwent surgical repair within 30 days postinjury and were followed up for 3 months or more postoperative (mean follow-up period: 8.4 months, range: 3-59 months). Orbital blowout fractures were classified into one of three shapes: (1) trap-door fracture with muscle entrapment, (2) trap-door fracture with incarcerated tissue and (3) depressed fragment fracture. Ocular motility was estimated by percentage of Hess area ratio $(H A R \%)$ on the Hess chart at the final follow-up examination. In addition, correlations between postinjury surgical timing and HAR\% were analysed.

Results The mean postinjury surgical timing was $10.7 \pm 7.8$ days (range: $0-30$ days). The mean postoperative HAR\% $(92.9 \% \pm 10.5 \%)$ was significantly improved compared with preoperative HAR\%

$(73.5 \% \pm 21.7 \%)(p<0.01)$. The mean postoperative HAR\% (98.3\% $4.4 \%)$ of the orbital-floor trap-door fracture patients with incarcerated tissue who underwent surgical repair within 8 days postinjury was significantly better than that of the patients who underwent surgical repair after 8 days $(94.2 \% \pm 5.8 \%)(p<0.01)$.

Conclusions Patients with orbital-floor trap-door blowout fractures with incarcerated tissue that were repaired within 8 days postinjury had better outcomes than those repaired after 8 days, and HAR\% is a useful method to record orbital fracture surgical outcomes.

\section{INTRODUCTION}

Orbital blowout fractures were first described in 1957 by Smith and Regan, who reported that they are caused by a sudden increase in intraorbital pressure. ${ }^{1}$ Blunt trauma to the orbit commonly causes fractures of the orbital floor and/or medial wall. In such cases, there may be trapping or prolapse of orbital soft tissue, and extraocular muscle dysfunction may cause diplopia and ocular motility disturbances.

The surgical timing for the repair of orbital fractures is a topic that is still controversial.Ophthalmologists and many surgical specialists including otolaryngologists, maxillofacial specialists and plastic surgeons treat orbital blowout fractures, so there has not been the common consensus of evaluation of the surgical results. One of the goals of orbital fracture repair is normalisation of ocular motility, and the other is the prevention/management of enophthalmos. Thus, objective methods are needed to evaluate ocular motility presurgery and postsurgery.

Numerous previous studies emphasised the presence of diplopia, but most of those reports did not refer to the objective data of ocular motility. To evaluate ocular motility objectively, we used the Hess screen test, designed by Walter Rudolf Hess in $1908,{ }^{2}$ which helped us to monitor changes in ocular motility of patients with orbital fractures. In cases of orbital fracture, ocular muscle dysfunction within 30 degrees can easily be identified. Furuta et al reported that they evaluated diplopia using the percentage of Hess area ratio (HAR\%) based on the Hess chart. ${ }^{3}$ In addition, Pier et al reported that HAR\% was effective in predicting postoperative diplopia in 30 patients undergoing surgical repair for orbital blowout fractures. ${ }^{4}$ The aim of this present study was to elucidate the best timing for surgical repair of orbital blowout fractures by use of HAR \% for objective evaluation of ocular motility.

\section{METHODS \\ Subjects}

In this retrospective study, 197 consecutive patients (154 males and 43 females; mean age: 29.0 years (range: 7-85 years)) with orbital fracture were included. All patients were diagnosed with orbital fracture at the Department of Ophthalmology, Kyoto Prefectural University of Medicine, Kyoto, Japan, between January 2007 and August 2015. All patients underwent surgical repair within 30 days postdiagnosis and were followed up for 3 months or more postoperative (mean follow-up period: 8.4 months, range: 3-59 months).

\section{Instrumentation used for examination}

In this study, images were obtained using a multislice CT machine (Aquilion RXL; Toshiba Medical Systems Corporation, Tokyo, Japan) and corresponding software, with bone-tissue (level $450 \mathrm{HU}$, width $2500 \mathrm{HU}$ ) and soft-tissue (level $100 \mathrm{HU}$, width $300 \mathrm{HU}$ ) windows and a slice thickness of $1.0 \mathrm{~mm}$. Hess screen tests using an Electronic Hess Screen (Haag-Streit UK, Clement Clarke International, Essex, UK) and a Hess chart (Handaya, Tokyo, Japan) were performed on all patients at the initial examination and at each postoperative follow-up examination, with the Hess chart being used to calculate the HAR\% (figure 1).

The exclusion criteria in this study included patients who underwent surgical repair of the fractures at another hospital and patients who 


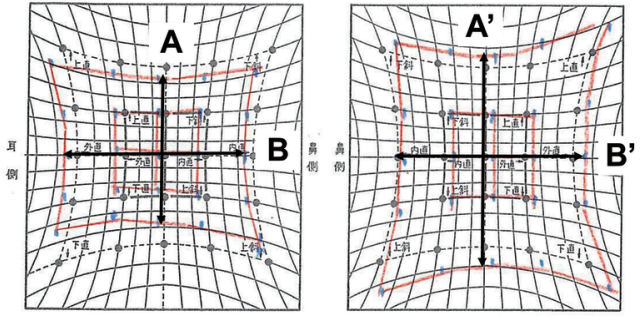

HAR $\%=\frac{\text { Affected side (vertical } \times \text { horizontal }) \mathrm{mm}^{2}}{\text { Healthy side (vertical } \times \text { horizontal }) \mathrm{mm}^{2}} \times 100(\%)$

Figure 1 Images showing how the Hess area ratio (HAR\%) is calculated: affected side length between vertical plot: $A(\mathrm{~mm})$, length between horizontal plot: $B(\mathrm{~mm})$. Healthy side length between vertical plot: $A^{\prime}(\mathrm{mm})$, length between horizontal plot: $B^{\prime}(\mathrm{mm}) . H A R \%$ is calculated using the following formula: $100 \times(A \times B) /\left(A^{\prime} \times B^{\prime}\right)(\%)$.

underwent surgical repair more than 1 month postinjury. In addition, patients who had severe eyelid swelling and intraorbital emphysema at the initial examination were also excluded, due to the fact that severe eyelid swelling disturbs normal ocular motility and makes it difficult to obtain correct Hess screen test measurements.

In all patients, the CT images were reviewed by an experienced oculoplastic surgeon (AW) to confirm the diagnosis of orbital fracture. Fractures were classified by region and shape via the multislice CT images, with the regions being classified as orbital floor, medial wall or combined orbital floor and medial wall. Shapes were classified as trap-door fracture with muscle entrapment, trap-door fracture with incarcerated tissue or depressed fragment fracture, in accordance with the classifications described by Beigi et $a l^{5}$ (figure 2). Surgical indications were determined by the presence of symptoms, such as diplopia, pain with ocular movement and limitation of ocular motility.

Two different surgical techniques were used for orbital reconstruction, that is, subciliary transcutaneous incision for orbital floor fractures and medial transcutaneous incision (ie, the Lynch incision) for medial wall fractures. In order to secure a wide surgical field, we used the transcutaneous incision for the orbital floor fractures, as it is necessary to confirm all edges of the fracture site in order to reverse the prolapse orbital soft tissues to the orbit and to insert the reconstruction material; the transcutaneous incision also makes it more easily for us to repair the fractures. All operations were conducted by two experienced oculoplastic surgeons (YY and AW).

For the orbital wall reconstructions, an unsintered hydroxyapatite particles/poly L-lactide composite device (OSTEOTRANS MX; Takiron, Tokyo, Japan), a silicone silastic sheet (Eyeball

\section{Classification of floor blow- out fracture}

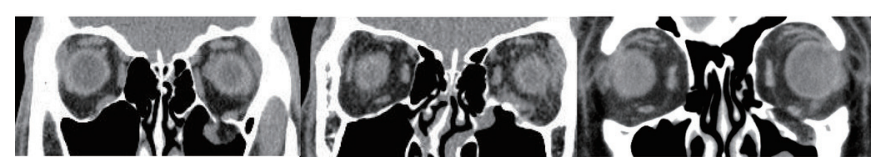

Figure 2 Corneal CT scan image demonstrating an isolated orbital floor trap-door fracture with incarcerated tissue (left), trap-door fracture with muscle entrapment (middle) and depressed floor-fragment fracture (right).
Table 1 Profiles of the 197 patients with orbital blowout fractures

\begin{tabular}{lcccc}
\hline & $\begin{array}{l}\text { Orbital } \\
\text { floor }\end{array}$ & $\begin{array}{l}\text { Medial } \\
\text { wall }\end{array}$ & $\begin{array}{l}\text { Orbital floor and } \\
\text { medial wall }\end{array}$ & Total \\
\hline $\begin{array}{l}\text { Trap-door fracture with muscle } \\
\text { entrapment }\end{array}$ & 3 & 1 & 0 & 4 \\
$\begin{array}{l}\text { Trap-door fracture with } \\
\text { incarcerated tissue }\end{array}$ & 51 & 6 & 3 & 60 \\
Depressed fragment fracture & 91 & 22 & 20 & 133 \\
Total & 145 & 29 & 23 & 197 \\
\hline
\end{tabular}

restraint insert; KOKEN, Tokyo Japan) and hydroxyapatite (Apaceram; HOYA, Tokyo, Japan) were used. The specific materials used for each orbital fracture reconstruction was selected by the surgeon at the time of operation in each case.

\section{Statistical analysis}

The Mann-Whitney U test was used to compare HAR\% between the preoperative and postoperative outcomes. Mann-Whitney $U$ test $p$ values were used for statistical significance, with a $p$ value of $<0.05$ considered statistically significant. This study was approved by the Institutional Review Board and Ethics Committee of Kyoto Prefectural University of Medicine, and prior informed consent was obtained from all patients involved in the study.

\section{RESULTS}

\section{Patient characteristics}

Of the 197 total cases, 145 patients (73.6\%) had orbital floor fractures, 29 (14.7\%) had orbital medial wall fractures and 23 (11.7\%) had combined orbital medial wall and floor fractures. In addition, 133 patients had orbital depressed fragment fractures, 60 patients had orbital trap-door fracture with incarcerated tissue and 4 patients had orbital trap-door fracture with muscle entrapment. The profiles of the 197 patients with orbital blowout fractures of each region and shape are shown in table 1.

The mean elapsed time from initial injury to subsequent surgery was $10.7 \pm 7.8$ days (range: $0-30$ days). The mean postoperative $\mathrm{HAR} \%$ at final follow-up $(92.9 \% \pm 10.5 \%)$ was significantly better than the mean preoperative HAR\% $(73.5 \% \pm 21.7 \%)$ $(\mathrm{p}<0.01)$ (figures 3 and 4). Of the 197 total cases, 175 patients

\section{Record of HAR\% Post Surgery}

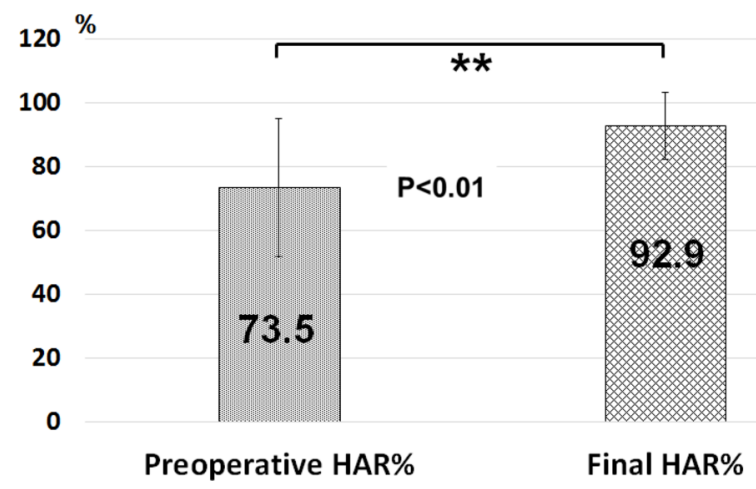

Figure 3 Images showing improvement of HAR\% postsurgery. Ocular motility was evaluated by HAR\% prior to surgery (left) and at the final follow-up examination (right). HAR, Hess area ratio. 


\section{Regions and shapes}
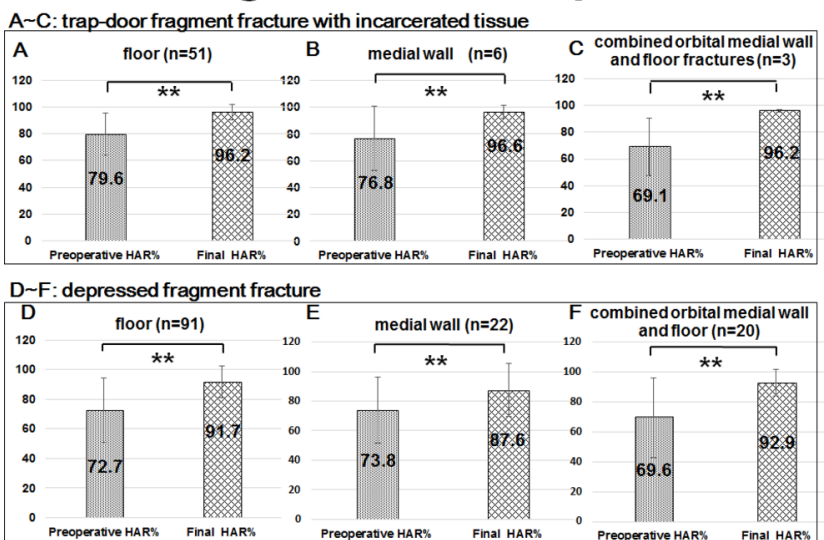

Figure 4 (A-C) Images showing improvement of HAR\% postsurgery. Preoperative (left) and final follow-up (right) of HAR\% of trap-door fragment fracture with incarcerated tissue. (D-F) Images showing improvement of HAR\% postsurgery. Preoperative (left) and final followup (right) of HAR\% of the depressed fragment fracture. HAR, Hess area ratio.

(88.8\%) attained better HAR\%, 2 (1\%) had the same HAR\% and $20(10.2 \%)$ attained worse HAR\%.

Of the 24 cases who had the same or had attained worse HAR\%, four patients had high preoperative and postoperative HAR (ie, preoperative and postoperative HAR >100\%). None of the 22 improved cases (mean preoperative HAR\%: $90.1 \% \pm 18.9 \%)$ were significantly better than the 175 improved cases (mean preoperative HAR\%: 71.4\% $\pm 21.2 \%)(\mathrm{p}<0.001)$.

CT-scan images demonstrating the preoperative depressed floor-fragment fracture (left) and the surgical repair at 1 day postoperative (right) are shown in figure 5.

\section{Association between surgical timing and final HAR\%}

The mean HAR\% $(98.3 \% \pm 4.4 \%)$ of the patients with orbital floor trap-door fracture with incarcerated tissue who underwent surgical repair within 8 days postinjury was significantly better than that of the final follow-up HAR\% $(94.2 \% \pm 5.8 \%)$ of the patients who underwent surgical repair more than 8 days postinjury $(\mathrm{p}<0.01)$. However, the mean preoperative HAR\% $(74.9 \% \pm 22.5 \%)$ of the patients with orbital floor trap-door fracture with incarcerated tissue who underwent surgical repair within 8 days postinjury was not significantly better than that of the preoperative HAR\% $(78.4 \% \pm 16.2 \%)$ of the patients who underwent surgical repair more than 8 days postinjury $(p>0.05)$.

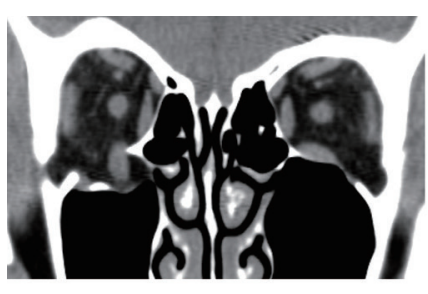

Preoperative

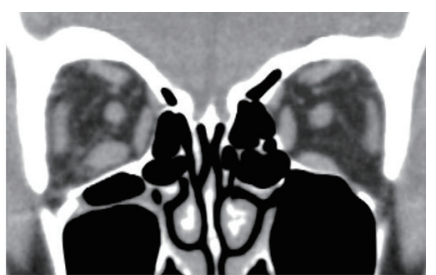

After surgical repair
Figure 5 Corneal CT scan images demonstrating a preoperative depressed floor-fragment fracture (left) and the same case on the day after surgical repair (right).
No significant difference was found in the mean final follow-up HAR\% of the patients with either depressed floor-fragment fracture or depressed medial-fragment fracture and combined depressed floor and medial wall-fragment fracture who underwent surgical repair within 1 month postinjury.

This study included three orbital-floor trap-door fracture patients and one patient with medial-wall trap-door fracture with muscle entrapment. All four patients who had trap-door fractures with muscle entrapment underwent surgical repair within 6 days postinjury, and the mean final follow-up HAR\% $(100.8 \% \pm 8.0 \%)$ was significantly better than that of the initial HAR\% (30.4\% $\pm 9.0 \%)(\mathrm{p}<0.01)$.

The total operation time was within 3 hours for all of the orbital floor fractures and orbital medial wall fractures. In addition, the total operation time was within 3.5 hours for all of the combined orbital floor and medial wall fracture. In all patients, there were no intraoperative complications. However, it should be noted that some postoperative complications were observed at the mean follow-up period of 8.4 months. Postoperative infraorbital nerve dysfunction for dysaesthesia was observed in four patients, and orbital haematoma was observed in two patients. Those two patients complained of postoperative diplopia and severe eyelid swelling 2 days after surgery, and a subsequent CT scan revealed the orbital haematoma. However, the haematomas did not enlarge in those two patients, and CT imaging revealed that they had disappeared at 1 month postoperative and both patients stopped complaining of diplopia and pain.

Association between reconstruction implant and final $\mathrm{HAR} \%$ Orbital blowout fractures were reconstructed using the OSTEOTRANS MX unsintered hydroxyapatite particles/poly L-lactide composite device in 120 patients (61\%), a silicone silastic sheet in 50 patients (25\%), a combination of OSTEOTRANS MX and silicone silastic sheet in five patients (3\%), a combination of silicone silastic sheet and hydroxyapatite in 16 patients $(8 \%)$, or nothing in six patients (3\%). Before OSTEOTRANS MX became commercially available, depressed fragment fractures were traditionally reconstructed using unsintered hydroxyapatite particles and the silicone silastic sheet. Now that OSTEOTRANS MX has become available, it is used for the reconstruction of nearly all orbital blowout fractures. Now, only in cases where the fracture site cannot be covered completely are the fractures reconstructed via a combination of OSTEOTRANS MX and a silicone silastic sheet. Regardless of the reconstruction implant material used, there was no significant difference of final follow-up HAR\%. A comparison of the reconstruction implant used for the blowout fracture subjects is shown in table 2 .

\section{Association between age and final HAR\%}

Following the method previously described by Egbert et al, ${ }^{6}$ the patients were classified into one of the following two groups: $(1)<18$ years of age $(n=70)$ and $(2) \geq 18$ years of age $(n=128)$. In the patients $<18$ years old, the mean postoperative HAR $\%$ $(96.9 \% \pm 5.5 \%)$ was significantly improved compared with preoperative HAR\% $(77.5 \% \pm 18.0 \%)(\mathrm{p}<0.01)$. In the patients $\geq 18$ years old, the mean postoperative HAR\% (90.7\% $111.9 \%)$ was also significantly improved compared with preoperative HAR\% $(71.3 \% \pm 23.3 \%) \quad(\mathrm{p}<0.01)$. However, in the patients $<18$ years old, the mean postoperative HAR\% $(96.9 \% \pm 5.5 \%)$ was significantly better than that of the patients $\geq 18$ years old $(90.7 \% \pm 11.9 \%)(\mathrm{p}<0.01)$. The age comparison data are shown in table 3 . 
Table 2 Detail of reconstruction implant

\begin{tabular}{llllll}
\hline & Osteotrans & Silastic sheet & Osteotrans and silastic sheet & Silastic sheet and hydroxyapatite & Nothing \\
\hline No. of cases & 120 & 50 & 5 & 16 & 13 \\
Day for surgical repair & 9.8 & 11.6 & $72.5 \pm 22.2$ & $72.4 \pm 23.5$ & 6 \\
Preoperative HAR\% & $74.1 \pm 21.8$ & $71.5 \pm 22.5$ & $92.5 \pm 6.9$ & $89.7 \pm 17.2$ & $83.6 \pm 7.1$ \\
\hline Final HAR\% & $93.3 \pm 10.6$ & $93.2 \pm 8.0$ & & $92.5 \pm 6.8$ \\
\hline
\end{tabular}

HAR, Hess area ratio.

\section{DISCUSSION}

Orbital blowout fractures may cause serious complications such as diplopia and enophthalmos, which may be caused by the entrapment of the extraocular tissues, muscles or fat within the fractured bone or the swelling of these intraorbital tissues. ${ }^{7}$ Burm et $a l^{8}$ reported that in 82 patients, $25 \%$ of the medial wall fractures, $80 \%$ of the orbital floor fractures and $80.9 \%$ of the combined orbital floor and medial wall fractures were associated with diplopia. They also reported that limitation of eye movement was observed in $12.5 \%$ of medial wall fractures, $73.3 \%$ of floor fractures and $47.6 \%$ of combined orbital floor and medial wall fractures.

In two previous reports, the effect of the surgical repair was only determined by the presence or absence of diplopia. ${ }^{8} 9$ However, the patient's complaints of diplopia in those studies were too subjective to compare the preoperative and postoperative repair. The objective methods used to evaluate diplopia were a binocular single vision test (BSV) and a Hess screen test. Alhamdani et $a l^{10}$ reported that improvement of the BSV score in the patients with surgical repair was not found to be significantly correlated with subjective outcome in relation to diplopia. In this study, we used the Hess screen test to evaluate ocular motility. This test helps us easily to monitor changes in ocular motility in patients with orbital blowout fractures. In a previous report from Furuta et al, HAR\% was the objective index to compare the surgical outcomes in patients with orbital blowout fractures. ${ }^{3}$ Pier et al reported that HAR\% was effective for predicting postoperative diplopia in 30 patients undergoing surgical repair for orbital blowout fractures. ${ }^{4}$ In those reports, all patients with preoperative HAR $>85 \%$ had no postoperative diplopia. In our present study, the mean final follow-up HAR\% $(92.9 \% \pm 10.5 \%)$ was significantly better than that of the initial HAR\% $(73.5 \% \pm 21.7 \%)(\mathrm{p}<0.01)$. The final HAR $\%$ in 175 patients $(88.8 \%)$ improved with surgical repair. Our results show that the appropriate surgical repair improved HAR $\%$ and that changes in ocular motility were easily monitored by HAR\%.

It should be noted that the surgical repair resulted in poor improvement of HAR\% in $22(11.2 \%)$ of the 197 patients. Of the 22 cases that attained a worse HAR\%, two cases had the same HAR\%, three cases who had a preoperative HAR of $<55 \%$ had poor improvement of HAR\% at final examination, 14 patients (63.6\%) had orbital floor fractures and 8 (36.4\%) had orbital

\begin{tabular}{lll}
\hline Table 3 & Age comparison data & \\
\hline & $\begin{array}{l}\text { Patients }<18 \text { years } \\
\text { of age }\end{array}$ & $\begin{array}{l}\text { Patients } \geq 18 \text { years } \\
\text { of age }\end{array}$ \\
\hline No. of cases & 70 & 128 \\
\hline Mean age & $14.2 \pm 2.7$ & $37.2 \pm 17.9$ \\
Day for surgical repair & 10.3 & 10.9 \\
Preoperative HAR\% & $77.5 \pm 18.0$ & $71.3 \pm 23.3$ \\
Final HAR\% & $96.9 \pm 5.5$ & $90.7 \pm 11.9$ \\
\hline
\end{tabular}

HAR, Hess area ratio. medial wall fractures. In addition, 15 of the 22 cases had orbital depressed fragment fractures, and 7 cases had orbital trap-door fracture with incarcerated tissue. The mean preoperative HAR $\%$ of the 22 cases with poor improvement $(90.1 \% \pm 18.9 \%)$ was significantly better than that of the 175 cases with improvement $(71.4 \% \pm 21.2 \%)(p<0.001)$. This finding indicates that the preoperative HAR $\%$ in the 22 patients with poor improvement was not severely poor, even though the degree of improvement was poor. Additional possible reasons for those poor results might have been severe displacement of orbital soft tissue and muscle by trauma. With depressed floor-fragment fracture, especially a large size depressed floor-fragment, prolapsed orbital soft tissue and muscle cannot necessarily correlate with a fully normal functional movement. ${ }^{11}$ Koornneef reported that the orbital soft tissues are interconnected by a network known as the connective tissue septal system. ${ }^{12}$ The surgical repair may cause distortion of this network, and thus affect postoperative ocular motility.

Surgical timing of orbital blowout fracture repair is a subject that remains controversial. Burnstine ${ }^{13}$ reported that surgery performed within 2 weeks postinjury is recommended in cases of symptomatic diplopia with positive forced ductions and that evidence of orbital soft tissue entrapment on CT examination or large orbital floor fractures may cause latent enophthalmos or hypo-ophthalmos. In two other previous reports, it was recommended that early surgical repair results in rapid improvement of ocular mortality and diplopia. ${ }^{14} 15$ However, Simon et al ${ }^{16}$ reported that the outcome of two groups were equivalent in their study that compared early surgery (20 patients treated within 2 weeks postinjury) with delayed surgery (30 patients treated after 2 weeks postinjury). Moreover, Scawn et $a l^{17}$ reported that delay of surgical repair beyond 6 weeks postinjury can also improve diplopia and enophthalmos.

It should be noted that when we encounter patients with orbital fractures who have poor symptoms, we sometimes opt for no surgical repair. Alhamdani et $a l^{10}$ reported the improvement of the BSV score in some patients with no surgery within 2 weeks. In that study, they stated that in orbital fracture patients with a high BSV, a waiting period with strict observation is recommended prior to performing surgery. Kasaee et al ${ }^{18}$ reported that in 72 patients with orbital fractures, only one patient $(1.4 \%)$ had diplopia after 6 months observation.

It should be noted that there are some reasons regarding the differences of surgical timing in the above-cited reports. Some of the reports considered only the time for surgical repair postinjury, yet not the region and shape of the fractures. Recently, CT allows for a more discriminating evaluation of bone/muscle/soft tissue in orbital blowout fractures than was previously available for surgeons. CT images allow for the region and shapes to be easily detected and then classified. ${ }^{5}$

There is a strong consensus in regard to the timing of surgical repair for a better functional outcome in the trapdoor fracture with muscle entrapment. Surgical repair within 


\section{Recommended surgical repair within 8 days after injury}

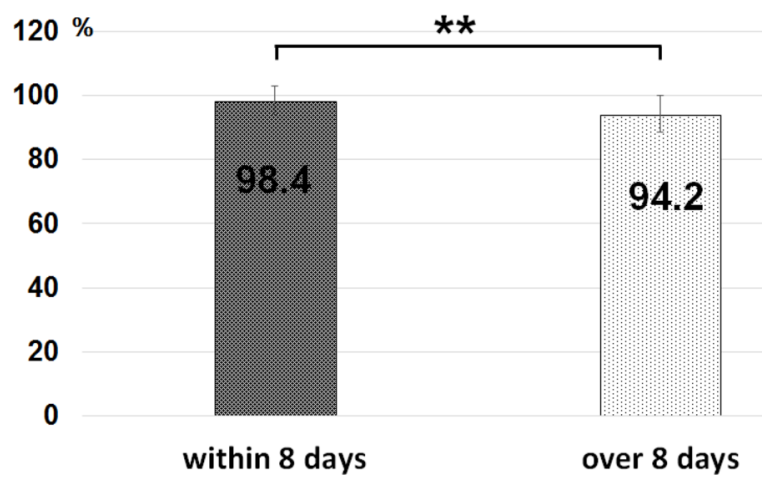

Figure 6 Images showing final follow-up HAR\% of a patient with orbital floor trap-door fracture with incarcerated tissue. The mean final follow-up HAR\% $(98.3 \% \pm 4.4 \%)$ of the patient who underwent surgical repair within 8 days postinjury (left) was significantly better than the final follow-up HAR\% $(94.2 \% \pm 5.8 \%)$ of the patient who underwent surgical repair after 8 days postinjury (right) $(p<0.01)$. HAR, Hess area ratio.

48 hours postinjury is recommended for paediatric 'white-eyed blowout' fractures. ${ }^{13}$ According to the recommendations stated in previous reports, we performed the surgical repair in four patients with trap-door fracture with muscle entrapment as soon as possible. In those four cases, the mean final follow-up HAR\% $(100.8 \% \pm 8.0 \%)$ was significantly better than that of the initial HAR\% $(30.4 \% \pm 9.0 \%)(\mathrm{p}<0.01)$. We also performed an early surgical repair of trap-door fracture with incarcerated tissue.

However, there is no consensus in regard to the timing of surgical repair in patients with orbital floor trap-door fracture with incarcerated tissue. In this study, the mean final follow-up HAR\% $(98.3 \% \pm 4.4 \%)$ in this type of patient who underwent surgical repair within 8 days postinjury was significantly better than the final HAR\% $(94.2 \% \pm 5.8 \%)$ in the patient who underwent surgery after 8 days postinjury $(p<0.01)$ (figure 6$)$. Patients with orbital-floor trap-door blowout fractures with incarcerated tissue that were repaired within 8 days postinjury had better outcomes than those repaired after 8 days. Thus, our findings might support early surgical repair within 8 days postinjury for the patients with orbital floor trap-door fracture with incarcerated tissue.

There was no significant difference of the mean final follow-up HAR\% of the patients by any surgical timing with either depressed floor-fragment fracture or depressed medial wall-fragment fracture and combined orbital floor, or medial wall fracture who underwent surgical repair within 1 month postinjury. We theorise that this result might be due to the fact that the surgical results in the patients who underwent treatment within 30 days were not significantly influenced by the surgical timing. Thirty days might not be a late timing and also be effective. Simon $e t a l^{16}$ compared the two groups via the enophthalmos and limitation of ocular motility and reported that there were no significant differences in surgical outcome between early (ie, within 2 weeks postinjury) and late surgical repair.

In addition, there was no significant difference of the mean final follow-up HAR\% of the patients by each region with either a trap-door fracture with incarcerated tissue or a depressed fragment fracture. The HAR\% of a patient with a depressed medial wall-fragment fracture tended towards less improvement than that of a patient with a depressed floor-fragment fracture. However, the final HAR $\%$ of a patient with a depressed medial wall-fragment fracture and a depressed floor-fragment fracture were significantly better than that of the preoperative HAR $\%$ $(\mathrm{p}<0.01)$.

Moreover, the material selected for orbital wall reconstruction may influence the recovery of ocular motility. We compared the surgical results of three reconstruction materials by HAR\%. However, there was no significant difference of improvement, regardless of implant material used (table 2).

It is important to note that this study did have several limitations. Examination of the preoperative Hess screen test was changeable by the timing postinjury. In addition, this examination displays ocular motility within 30 degrees, yet it cannot express the limitation over 30 degrees. An improved type of examination that can illustrate the patient's diplopia more objectively is needed in the future.

In conclusion, the findings of this study clearly demonstrate that the distinct region and shape of orbital fractures might be effectively analysed via the use of HAR $\%$ and that both the region and shape of orbital fractures affect the surgical outcome. These findings showed that patients with orbital fractures obtained good ocular movement via surgery and that HAR\% was an instructive and useful method to record and evaluate the surgical outcomes in patients with orbital fracture.

Acknowledgements The authors wish to thank John Bush for his excellent and thorough review of the manuscript.

Contributors All persons who meet authorship criteria are listed as authors, and all authors certify that they have participated sufficiently in the work and take personal responsibility for the content, including participation in the concept, design, analysis, writing and/or revision of the manuscript. Furthermore, each author certifies that this material, or similar material, has not been, and will not be, submitted to or published in any other publication before its appearance in the British Journal of Ophthalmology. Conception and design of study: YY and AW. Acquisition of data: YY and AW. Analysis and/or interpretation of data: YY and AW. Drafting of the manuscript: AW, CS and SK. Critical revision of the manuscript for important intellectual content: AW, CS and SK. Approval of the version of the manuscript to be published: YY, AW, CS and SK.

Competing interests None declared.

Patient consent Obtained.

Ethics approval Institutional Review Board of Kyoto Prefectural University of Medicine.

Provenance and peer review Not commissioned; externally peer reviewed.

Open Access This is an Open Access article distributed in accordance with the Creative Commons Attribution Non Commercial (CC BY-NC 4.0) license, which permits others to distribute, remix, adapt, build upon this work non-commercially, and license their derivative works on different terms, provided the original work is properly cited and the use is non-commercial. See: http://creativecommons.org/ licenses/by-nc/4.0/

(C) Article author(s) (or their employer(s) unless otherwise stated in the text of the article) 2018. All rights reserved. No commercial use is permitted unless otherwise expressly granted.

\section{REFERENCES}

1 Smith B, Regan WF. Blow-out fracture of the orbit; mechanism and correction of internal orbital fracture. Am J Ophthalmol 1957;44:733-9.

2 Hess WR. Ein einfaches messendes Verfahren zur Motilitätsprüfung der Augen. Zeitschrift für Augenheilkunde 1916;15:201-19.

3 Furuta M, Yago K, lida T. Correlation between ocular motility and evaluation of computed tomography in orbital blowout fracture. Am J Ophthalmol 2006;142:1019-25.

4 Grenga PL, Reale G, Cofone C, et al. Hess area ratio and diplopia: evaluation of 30 patients undergoing surgical repair for orbital blow-out fracture. Ophthal Plast Reconstr Surg 2009;25:123-5.

5 Beigi B, Khandwala M, Gupta D. Management of pure orbital floor fractures: a proposed protocol to prevent unnecessary or early surgery. Orbit 2014;33:336-42. 
6 Egbert JE, May K, Kersten RC, et al. Pediatric orbital floor fracture: direct extraocular muscle involvement. Ophthalmology 2000;107:1875-9.

7 Jin HR, Shin SO, Choo MJ, et al. Relationship between the extent of fracture and the degree of enophthalmos in isolated blowout fractures of the medial orbital wall. J Oral Maxillofac Surg 2000;58:617-20.

8 Burm JS, Chung CH, Oh SJ. Pure orbital blowout fracture: new concepts and importance of medial orbital blowout fracture. Plast Reconstr Surg 1999:103:1839-49.

9 Hoşal BM, Beatty RL. Diplopia and enophthalmos after surgical repair of blowout fracture. Orbit 2002;21:27-33.

10 Alhamdani F, Durham J, Greenwood M, et al. Diplopia and ocular motility in orbital blow-out fractures: 10-year retrospective study. I Craniomaxillofac Surg 2015:43:1010-6.

11 Liao JC, Elmalem VI, Wells TS, et al. Surgical timing and postoperative ocular motility in type B orbital blowout fractures. Ophthal Plast Reconstr Surg 2015;31:29-33.
12 Koornneef L. Orbital septa: anatomy and function. Ophthalmology 1979; 86:876-80.

13 Burnstine MA. Clinical recommendations for repair of isolated orbital floor fractures: an evidence-based analysis. Ophthalmology 2002;109:1207-10.

14 Hawes MJ, Dortzbach RK. Surgery on orbital floor fractures. influence of time of repair and fracture size. Ophthalmology 1983;90:1066-70.

15 Egbert JE, May K, Kersten RC, et al. Pediatric orbital floor fracture : direct extraocular muscle involvement. Ophthalmology 2000;107:1875-9.

16 Simon GJ, Syed HM, McCann JD, et al. Early versus late repair of orbital blowout fractures. Ophthalmic Surg Lasers Imaging 2009:40:141-8.

17 Scawn RL, Lim LH, Whipple KM, et al. Outcomes of Orbital Blow-Out Fracture Repair Performed Beyond 6 Weeks after Injury. Ophthal Plast Reconstr Surg 2016:32:296-301.

18 Kasaee A, Mirmohammadsadeghi A, Kazemnezhad F, et al. The predictive factors of diplopia and extraocular movement limitations in isolated pure blow-out fracture. $J$ Curr Ophthalmol 2017;29:54-8. 